\title{
A MOBILE SYSTEM FOR REAL-TIME PATIENT-MONITORING WITH INTEGRATED PHYSIOLOGICAL SIGNAL PROCESSING
}

\author{
Han-Chang Wu ${ }^{1}$, Student Member, Chao-Hung Lin ${ }^{1}$, Kuang-Ching Wang ${ }^{1}$, Shao-Cheng Wang ${ }^{1}$, \\ Chien-Hao Chen ${ }^{1}$, Shuenn-Tsong Young ${ }^{2}$, and Te-Son Kuo ${ }^{1,3}$, Senior Member \\ ${ }^{1}$ Department of Electrical Engineering, National Taiwan University \\ ${ }^{2}$ Institute of Biomedical Engineering, National Yang-Ming University \\ ${ }^{3}$ Graduate Institute for Biomedical Engineering, National Taiwan University
}

\begin{abstract}
Telemedicine is a rapidly growing area and recently there are studies devoted to prehospital care of patients in emergency cases. Here we present a GSM-network-based, function-integrated telemonitoring system to give first aid before reaching the hospital.
\end{abstract}

Keywords: Telemedicine, GSM

\section{Introduction}

In order to provide complete management for the patient in emergency cases, we present a GSM-network-based, functionally integrated telemonitoring system to give first aid before reaching the hospital. The related studies point out that most of the cardiovascular or heavily wounded patients could have survived if immediate treatment were given. In our system, we acquire vital bio-signals (including ECG, respiration rate, heart rate and blood pressure) and still images of the patient, send these back to the hospital to get fast consultation and take care of the patient following the on-line instructions. All of these are going on under real time criterion using the integrated device we had developed. This system is low-cost, highly robust, easy to operate and have the potential to prevail.

\section{Methods}

The portable device is a fully-integrated system which allows telediagnosis and teleconsultation provided by experienced physicians. The system is based on client/server structure and features its GSM air interface link and collection of various vital bio-signals including ECG signals, oxygen saturation, heart rate and so on. The system also handles multimedia data such as still images, text, or graphical representation to make prescriptions as easy as possible.

We utilize the GSM data link as our air interface. The client side device gets on the GSM network to communicate with the server in the hospital via a notebook and a wireless modem (M1, Siemens). To ensure the reliability of the data transfer, we select the TCP/IP protocol, which performs error detection and flow control. TCP/IP is so popular that we can act as a node on the Internet and easily communicate with other nodes.

Despite the limited bandwidth in the current GSM system, our device features real-time data transmission by efficiently compress the large amount of ECG signal. We apply DWT (Discrete Wavelet Transform) to implement our compression scheme. The huge computation load has been taken care by a DSP processor thus we have multi-channel ECG signal and other important data as well.

Finally, we choose TMSC320C 32 DSP (Texas Instrument) to accomplish real-time compression and decompression. Also, the DSP-based hardware is able to collect the signals and transmit them to the notebook. Because of the amazing processing ability of DSP, we get a compression ratio of $4 \sim 10$ but still satisfy the real-time criterion. The ambulance personnel are allowed to adjust the compression rate arbitrarily to tradeoff between channel traffic and signal quality.resources to different tasks of different priority.

\section{Results}

We have developed a mobile device which realizes realtime telediagnosis and teleconsultation based on GSM network. All of the vital data of patients are collected by a DSP-based hardware, real-time compressed, and immediately sent back to the physicians in the hospital. Bilateral communication is also made possible. We believe the implementation of our system is a better way to provide a complete early management and will be helpful to rescue the patients.

\section{References}

(1) "A novel emergency telemedicine system based on wireless communication technology - AMBULANCE", IEEE Trans. Information Technology in Biomedicine, vol.2,no. 4, pp.261-267, Dec 1998

(2) Hajime Murakami, et. Al, "Telemedicine using mobile satellite communication," IEEE Trans. Biomedical Engineering, vol.41, pp.488-497, May 1994

(3) Marta J. Petersen, et. Al, "Telemedicine in Utah: The rural Utah telemedicine pilot project," IEEE FIE Proceedings, pp986-989, 1996 